\title{
Crescimento de Espécies Bioindicadoras do Residual do HERBICIDA (IMAZETHAPYR+IMAZAPIC), SEMEAdAS EM ROTAÇÃo COM ARRoZ Clearfield ${ }^{\circledR 1}$
}

\author{
Growth of Residual Herbicide (Imazethapyr+Imazapic) Bio-indicators Sown in Rotation with \\ Clearfield ${ }^{\circledR}$ Rice
}

\author{
SOUSA, C.P. ${ }^{2}$, BACARIN, M.A. ${ }^{3}$ e PINTO, J.J.O. ${ }^{4}$
}

\begin{abstract}
RESUMO - Neste trabalho, determinou-se o efeito residual do herbicida Only ${ }^{\mathbb{}}$ (imazethapyr+imazapic) sobre plantas de milho, pepino, rabanete e tomate, semeadas em solo no qual o herbicida foi aplicado há 1.100 dias. O estudo foi realizado em casa de vegetação, com o delineamento experimental de casualização por bloco, com quatro repetições por tratamento. Os tratamentos avaliados foram o residual do herbicida Only ${ }^{\circledR}$ aplicado sob as plantas de arroz CL na safra 2006/2007, nas doses de 0, 100, 150 e $200 \mathrm{~g}^{\text {ha-1 }}$ do produto comercial, acrescido de $0,5 \%$ do adjuvante Dash $^{\circledR}$. Foram semeadas sob as parcelas 15 sementes de cada espécie bioindicadora (milho, pepino, rabanete e tomate), sendo estas desbastadas para 10 plantas após a germinação. Após 60 dias da data da semeadura, foram avaliadas a altura de plantas, a massa seca da parte aérea e a massa seca das raízes, sendo esta última não realizada nas plantas de milho e de pepino. Os dados obtidos foram submetidos à análise da variância $(\mathrm{p} \leq 0,05)$ e testados por modelos de regressão polinomial. Conclui-se que houve presença de atividade residual da mistura comercial dos herbicidas (imazethapyr+imazapic) do grupo das imidazolinonas em solo após 1.100 dias da aplicação dos herbicidas. Isso indica que as plantas de milho, pepino, rabanete e tomate podem ser utilizadas como espécies bioindicadoras de atividade residual da mistura comercial dos herbicidas (imazethapyr+imazapic) e que o rabanete e o tomate são mais sensiveis à presença do produto, quando comparados ao milho e ao pepino.
\end{abstract}

Palavras-chave: Only ${ }^{\circledR}$, persistência, Oryza sativa, imidazolinonas.

ABSTRACT - This work aimed to determine the residual effect of the herbicide Only ${ }^{\circledR}$ (imazethapyr+imazapic) on corn, cucumber, radish and tomato grown in soil where the herbicide was applied 1.100 days earlier. The experiment was carried out in a greenhouse in a randomized block design, with four replicates per treatment. The treatments were the residual herbicide applied under Only ${ }^{\circledR}$ rice CL plants in the 2006/2007 crop season at doses of 0, 100, 150, and $200 \mathrm{~g} \mathrm{ha}^{-1}$ of the commercial product, plus $0.5 \%$ of adjuvant Dash ${ }^{\circledR}$. Fifteen seeds of each bio-indicator (corn, cucumber, radish and tomato) were sown in the plots and thinned to 10 plants after emergence. After 60 days of sowing, the variables plant height, aboveground dry biomass and root dry biomass were measured, except the latter for the corn plants. Data were subjected to analysis of variance $(p \leq 0.05)$ and tested by polynomial regression models. It was concluded that there was a residual activity of the herbicide Only (imazethapyr+imazapic) applied under greenhouse conditions, 1, 100 days after herbicide application DAA, negatively affecting plant height and aboveground dry biomass of corn, cucumber, radish and tomato. Thus, these species can be used as bio-indicators of residual activity of the commercial mixture of herbicides (imazethapyr + imazapic). Radish and tomato were more sensitive to the presence of the product, compared to corn and cucumber.

Keywords: Only ${ }^{\circledR}$, persistence, Oryza sativa, imidazolinones.

1 Recebido para publicação em 24.5.2011 e aprovado em 27.12.2011.

2 Engâ-Agrª. M.Sc., Doutoranda do Programa de Pós-Graduação em Fisiologia Vegetal, Laboratório de Metabolismo Vegetal, Dep. de Botânica, Instituto de Biologia, Universidade Federal de Pelotas - UFPEL, Campus Universitário S/N, 96160-000 Capão do LeãoRS, Brasil, <camilafepi@hotmail.com>; ${ }^{3}$ Engo-Agroo., Dr., Professor, Dep. de Botânica, Instituto de Biologia, UFPEL, <bacarin@ufpel.edu.br>; ${ }^{4}$ Engo--Agrº., Dr., Professor, Dep. de Fitossanidade, Faculdade de Agronomia, UFPEL, <jesuspinto@terra.com.br>. 


\section{INTRODUÇÃO}

Nas lavouras de arroz irrigado (Oryza sativa) do Rio Grande do Sul, a principal planta daninha encontrada é o arroz-vermelho, que possui diferentes ecótipos com características morfofisiológicas muito similares às do cultivado (Vaughan et al., 2001). Portanto, havia grande dificuldade de controle dessa espécie daninha nas lavouras de arroz irrigado, o que despertou pesquisas para o problema, as quais resultaram no desenvolvimento da tecnologia Clearfield $^{\mathbb{}}$. Essa tecnologia possibilita o controle químico do arroz-vermelho em lavouras de arroz cultivado, por meio do uso de herbicidas do grupo químico das imidazolinonas em cultivos com arroz tolerante. No Brasil, o herbicida inicialmente recomendado na tecnologia Clearfield ${ }^{\circledR}$ foi o Only $^{\circledR}$, composto pela mistura formulada dos herbicidas imazethapyr + imazapic, nas concentrações de 75 e 25 g i.a. $\mathrm{L}^{-1}$, respectivamente (Sosbai, 2010). Atualmente, foi lançado o herbicida Kifix $^{\circledR}$, composto pela mistura formulada dos herbicidas imazapyr + imazapic, nas concentrações de 525 e $175 \mathrm{~g}$ i.a. $\mathrm{kg}^{-1}$, respectivamente, também podendo ser utilizado nesse sistema.

Entre as principais caracteristicas dos herbicidas desse grupo está a prolongada atividade residual no solo (Loux \& Reese, 1993), cuja persistência atinge até 540 dias após a sua aplicação (Pinto et al., 2009). A persistência no solo é uma característica de alguns grupos químicos de herbicidas ou, ainda, de herbicidas específicos, podendo ser desejável ou não. Essa persistência é positiva quando resulta em periodo suficiente para manter a cultura livre da competição de plantas daninhas (Gazziero et al., 1997). Todavia, é indesejável quando resulta em injúrias para culturas em sucessão ou rotação ou mesmo pode causar aumento de riscos de contaminação ambiental.

A utilização de espécies não tolerantes pode ser comprometida caso o intervalo entre a aplicação de imazethapyr e a semeadura da cultura em rotação não seja observado (Williams et al., 2002). Nos EUA, onde a tecnologia Clearfield ${ }^{\circledR}$ foi desenvolvida, recomendase a utilização do herbicida imazethapyr por dois anos consecutivos, deixando o solo em repouso por, no mínimo, um ano. Apesar da orientação de estudos realizados no Brasil para que seja respeitada a mesma recomendação, muitos produtores acabam usando a tecnologia por mais de dois anos consecutivos. Além disso, deve-se considerar a diversidade de fatores relacionados à velocidade de degradação de um herbicida, o que dificulta estimar o tempo necessário para sua dissipação após a aplicação, uma vez que no ambiente existe um conjunto de condições e de variáveis que podem influenciar na decomposição do produto.

Além da utilização da tecnologia Clearfield $^{\circledR}$, a rotação do arroz irrigado com sorgo, milho ou soja também vem sendo empregada como técnica eficiente de manejo do arroz-vermelho, pois essas espécies requerem condições diferenciadas de manejo de água e solo e utilizam herbicidas diferentes dos aplicados no arroz - fatores que favorecem a redução da dominância de espécies concorrentes (Gomes Jr. \& Christoffoleti, 2008).

O objetivo deste trabalho foi determinar, por meio de bioensaio, o efeito residual da mistura comercial dos herbicidas imazethapyr+ imazapic nas plantas de milho, pepino, rabanete e tomate, semeadas 1.100 dias após a aplicação (DAA) dos herbicidas imazethapyr+ imazapic na cultura do arroz irrigado.

\section{MATERIAL E MÉTODOS}

O experimento foi realizado em casa de vegetação, com o delineamento experimental de casualização por bloco, com quatro repetições por tratamento. Foi avaliado o residual do herbicida Only ${ }^{\circledR}$, em um esquema de sucessão e rotação de culturas. As parcelas foram constituídas por caixas de polietileno $(60 \times 40 \times 20 \mathrm{~cm})$, cada uma contendo $48 \mathrm{dm}^{3}$ de solo, coletado em área de Planossolo Háplico Eutrófico solódico (Embrapa, 2006), a 10 cm de profundidade, cujas análises química e física encontram-se na Tabela 1 . No mês de setembro de 2006, foi realizada a semeadura do arroz, cv. IRGA $422 \mathrm{CL}$, utilizado na tecnologia Clearfield $^{\circledR}$. Quando as plantas de arroz se encontravam em estádio fenológico de três a quatro folhas, foram aplicados os tratamentos herbicidas, que corresponderam a 0, 100, 150 e $200 \mathrm{~g} \mathrm{ha}^{-1}$ da mistura comercial do herbicida Only ${ }^{\circledR}$, acrescidos do adjuvante $\operatorname{Dash}^{\circledR}$ a 
Tabela 1 - Diagnóstico para recomendação de calagem, adubação NPK, S, micronutrientes e relações molares do solo

\begin{tabular}{|c|c|c|c|c|c|c|c|c|c|c|c|}
\hline \multirow{3}{*}{$\begin{array}{l}\text { Registro } \\
\text { Amostra }\end{array}$} & \multirow{2}{*}{\multicolumn{2}{|c|}{ pH água }} & $\mathrm{Ca}$ & $\mathrm{Mg}$ & $\mathrm{Al}$ & $\mathrm{H}+\mathrm{Al}$ & CTC & \multicolumn{3}{|c|}{ Saturação (\%) } & \multirow{3}{*}{$\frac{\text { Índice SMP }}{6,3}$} \\
\hline & & & \multicolumn{5}{|c|}{$\left(\mathrm{cmol}_{\mathrm{c}} \mathrm{dm}^{-3}\right)$} & \multicolumn{2}{|l|}{$\mathrm{Al}$} & Bases & \\
\hline & \multicolumn{2}{|c|}{5,0} & 1,8 & 0,7 & 1,0 & 3,0 & 3,6 & \multicolumn{2}{|l|}{29} & 46 & \\
\hline Registro & \multicolumn{2}{|c|}{$\begin{array}{l}\text { \% Mat. Org. } \\
(\mathrm{m} / \mathrm{v})\end{array}$} & \multicolumn{2}{|c|}{$\%$ Argila } & Textura & \multicolumn{2}{|c|}{$\begin{array}{l}\text { P-Mehlich } \\
\left(\mathrm{mg} \mathrm{dm}^{-3}\right)\end{array}$} & P-Resin & \multicolumn{2}{|c|}{$\begin{array}{c}\text { CTC Ph } 7 \\
\left(\mathrm{cmol}_{\mathrm{c}} \mathrm{dm}^{-3}\right)\end{array}$} & $\begin{array}{c}\mathrm{K} \\
\left(\mathrm{mg} \mathrm{dm}^{-3}\right)\end{array}$ \\
\hline Amostra & \multicolumn{2}{|c|}{1,2} & \multicolumn{2}{|c|}{16} & 4,0 & \multicolumn{2}{|c|}{11,4} & --- & & 5,5 & 17 \\
\hline \multirow{2}{*}{ Registro } & $\mathrm{S}$ & $\mathrm{Cu}$ & $\mathrm{Zn}$ & B & $\mathrm{Fe}$ & $\mathrm{Mn}$ & $\mathrm{Na}$ & \multicolumn{4}{|c|}{ Relação } \\
\hline & \multicolumn{7}{|c|}{$\left(\mathrm{mg} \mathrm{dm}^{-3}\right)$} & $\mathrm{Ca} / \mathrm{Mg}$ & $\mathrm{Ca} / \mathrm{K}$ & $\mathrm{Mg} / \mathrm{K}$ & $\mathrm{K} /(\mathrm{Ca}+\mathrm{Mg})$ \\
\hline Amostra & --- & 0,9 & 1,0 & - & 13 & 17 & 17 & 2,5 & 43,6 & 17,8 & 0,027 \\
\hline
\end{tabular}

0,5\% v/v. A aplicação dos tratamentos foi feita com pulverizador costal pressurizado a $\mathrm{CO}_{2}$, com barra e dois bicos do tipo leque (110.02), proporcionando a aplicação de $150 \mathrm{~L} \mathrm{ha}^{-1}$ de líquido aspergido. Aos sete dias após a aplicação dos tratamentos, teve início a irrigação por inundação, sendo as parcelas mantidas com lâmina d'água de aproximadamente $4,0 \mathrm{~cm}$, até a maturação do arroz. Após a colheita, o solo não foi irrigado por um periodo de 45 dias, época em que foi semeada aveia-preta (Avena strigosa), em densidade de 300 sementes $\mathrm{m}^{2}$. A colheita da aveia foi feita quando as plantas se encontravam no estádio de dois e três afilhos. A seguir, o solo foi mantido sem irrigação até o início da próxima etapa, que consistiu da instalação, no mesmo substrato, em sequência, de dois experimentos com a cultura do milho (Zea mays). No primeiro experimento, o milho foi semeado na época correspondente à "safra", em setembro de 2007 , e no segundo se procedeu à semeadura do milho "safrinha", em fevereiro de 2008. As plantas, em ambos os casos, foram colhidas quando no tratamento testemunha se encontravam em estádio de quatro e cinco folhas. Após a colheita do milho, as caixas foram mantidas sem irrigação até novembro de 2008, quando foram sorteados e semeados quatro cultivares de arroz irrigado, em uma densidade de semeadura correspondente a $100 \mathrm{~kg} \mathrm{ha}^{-1}$. Aos sete dias após a semeadura, as parcelas foram mantidas com lâmina d'água de $4,0 \mathrm{~cm}$. A colheita ocorreu quando as plantas, nas parcelas testemunhas, encontravam-se em estádio V8. As caixas permaneceram sem irrigação até o início deste experimento na safra 2009/2010, onde foram semeadas sob as parcelas 15 sementes de cada espécie bioindicadora: milho, pepino (Cucumis sativus), rabanete (Raphanus sativus) e tomate (Solanum lycopersicum), sendo estas desbastadas para 10 plantas após a emergência. A irrigação foi mantida conforme a necessidade das plantas.

Foram determinadas a altura de plantas e a massa seca da parte aérea (MSA) de todas as espécies e a massa seca das raízes (MSR) somente do rabanete e do tomate. A altura das plantas bioindicadoras foi avaliada aos 60 dias após a semeadura, medindo-se a distância entre o colo da planta e o ápice da folha mais jovem. Após a coleta das plantas, a parte aérea e a raiz foram acondicionadas separadamente, em sacos de papel, e colocadas em estufa de circulação forçada, com temperatura de $50 \pm 5{ }^{\circ} \mathrm{C}$, até atingirem massa constante.

Os dados obtidos foram submetidos à análise da variância $(p \leq 0,05)$ e ajustados por modelos de regressão polinomial (Machado \& Conceição, 2007). A escolha dos modelos baseou-se na significância estatística (teste F), no ajuste do coeficiente de determinação $\left(R^{2}\right)$ e no significado biológico do modelo.

\section{RESULTADOS E DISCUSSÃO}

O efeito residual do herbicida $\mathrm{Only}^{\circledR}$ mostrou diferença significativa na altura de plantas, quando comparados os tratamentos 
utilizados, e os resultados relativos a essa variável ajustaram-se ao modelo linear utilizado (Figura 1). A altura de plantas, comparativamente ao tratamento testemunha, sofreu redução de 18, 38, 45 e 50\%, respectivamente para milho (Figura 1A), pepino, rabanete e tomate (Figura 1B), na presença de residual da dose de $100 \mathrm{~g} \mathrm{ha}^{-1}$ de imazethapyr+ imazapic. Entretanto, quando a dose utilizada foi de $200 \mathrm{~g} \mathrm{ha}^{-1}$, as reduções nesta variável aumentaram para 35, 77, 90 e 100\%, respectivamente, para essas mesmas espécies. Bond et al. (2006) já mencionavam o efeito do incremento da dose herbicida, resultando em aumento na fitotoxicidade, o que corrobora os resultados encontrados neste trabalho, em que foi observado que o incremento das doses de imazethapyr+imazapic reduziu drasticamente a altura das plantas de milho, rabanete, pepino e tomate. Pinto et al. (2009) também observaram redução no porte das plantas de milho semeado em rotação ao arroz CL, 540 dias após a aplicação dos herbicidas em sistema de sucessão/rotação.

No entanto, sabe-se que a persistência de herbicidas do grupo químico das imidazolinonas no solo é influenciada pelas propriedades deste, como o pH, a umidade, o teor de matéria orgânica e a textura (Stougaard et al., 1990).
A ação microbiana é o principal mecanismo de degradação desses herbicidas (Flint \& Witt, 1997) e pode acontecer em condições de aerobiose, como é o caso da degradação de imazethapyr constatada por Shaner \& O‘Connor, (1991), ou em condições de aerobiose e anaerobiose, no caso de imazapyr (Wang et al., 2006). Quando as condições ambientais favorecem o desenvolvimento dos microrganismos e a biodisponibilidade dos herbicidas, a degradação das imidazolinonas aumenta (Kraemer et al., 2009). Sabe-se que as condições de baixo teor de matéria orgânica (Tabela 1) e de períodos prolongados sem irrigação - em que o solo deste experimento foi mantido - provavelmente não favoreceram o desenvolvimento de microrganismos, o que pode justificar os resultados de persistência encontrados. Segundo Wang et al. (2006), a degradação de imazapyr é 2,3 a 4,4 vezes mais lenta em solos estéreis, quando comparada com a de solo em condições naturais.

A análise dos dados sobre a massa da matéria seca da parte aérea das espécies bioindicadoras (Figura 2) também mostrou diferença estatística significativa, sendo representada no modelo linear com declividade negativa. Comparando-se as aplicações de $100 \mathrm{~g} \mathrm{ha}^{-1} \mathrm{do}$ herbicida com o tratamento testemunha,
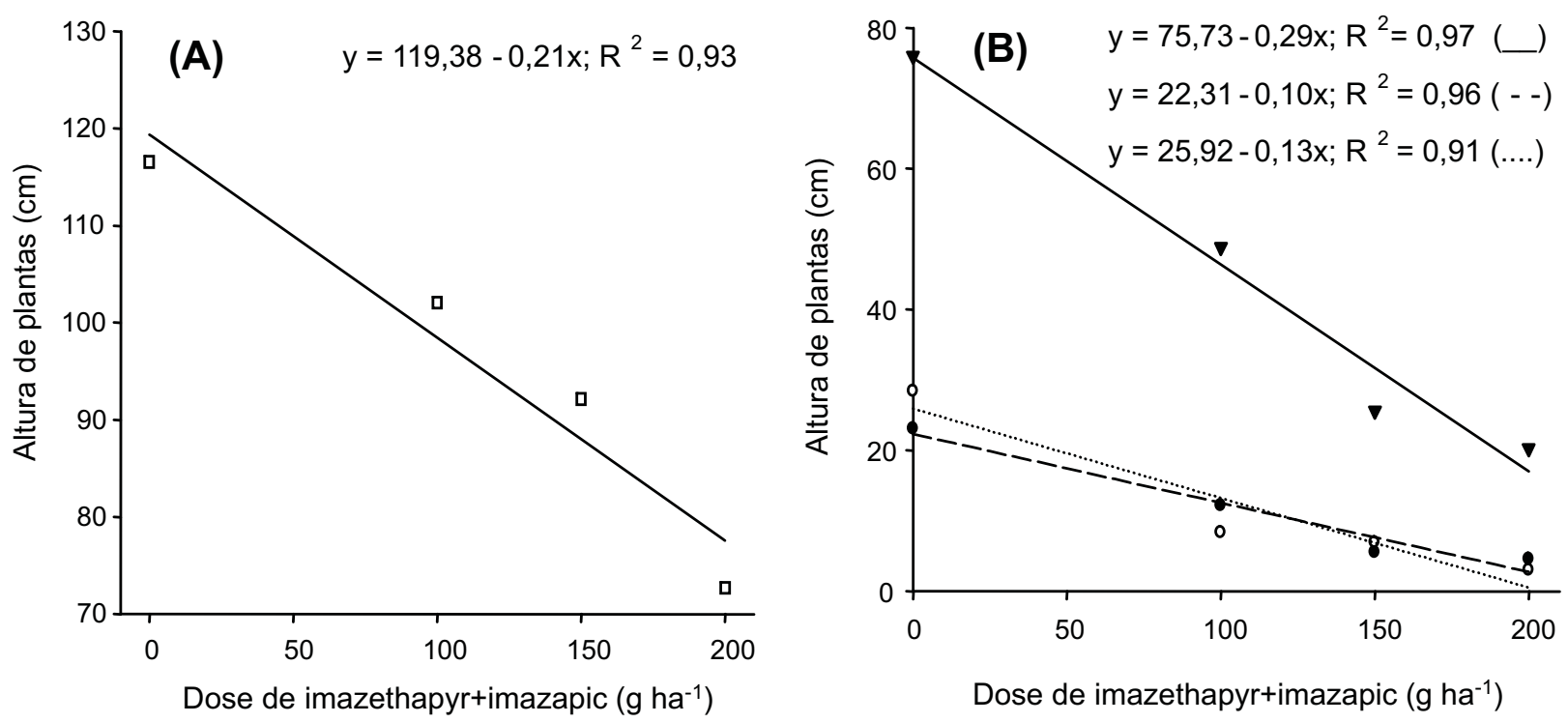

Figura 1 - Efeito residual do herbicida Only ${ }^{\circledR}$ na altura de plantas de milho (A e $\square$ ), pepino (_e $\boldsymbol{\nabla}$ ), rabanete (- - e •) e tomate (....e o) (B) aos 60 dias após a semeadura, cultivados em rotação com o arroz CL, aos 1.100 dias após a aplicação dos herbicidas. Capão do Leão - RS, 2010. 

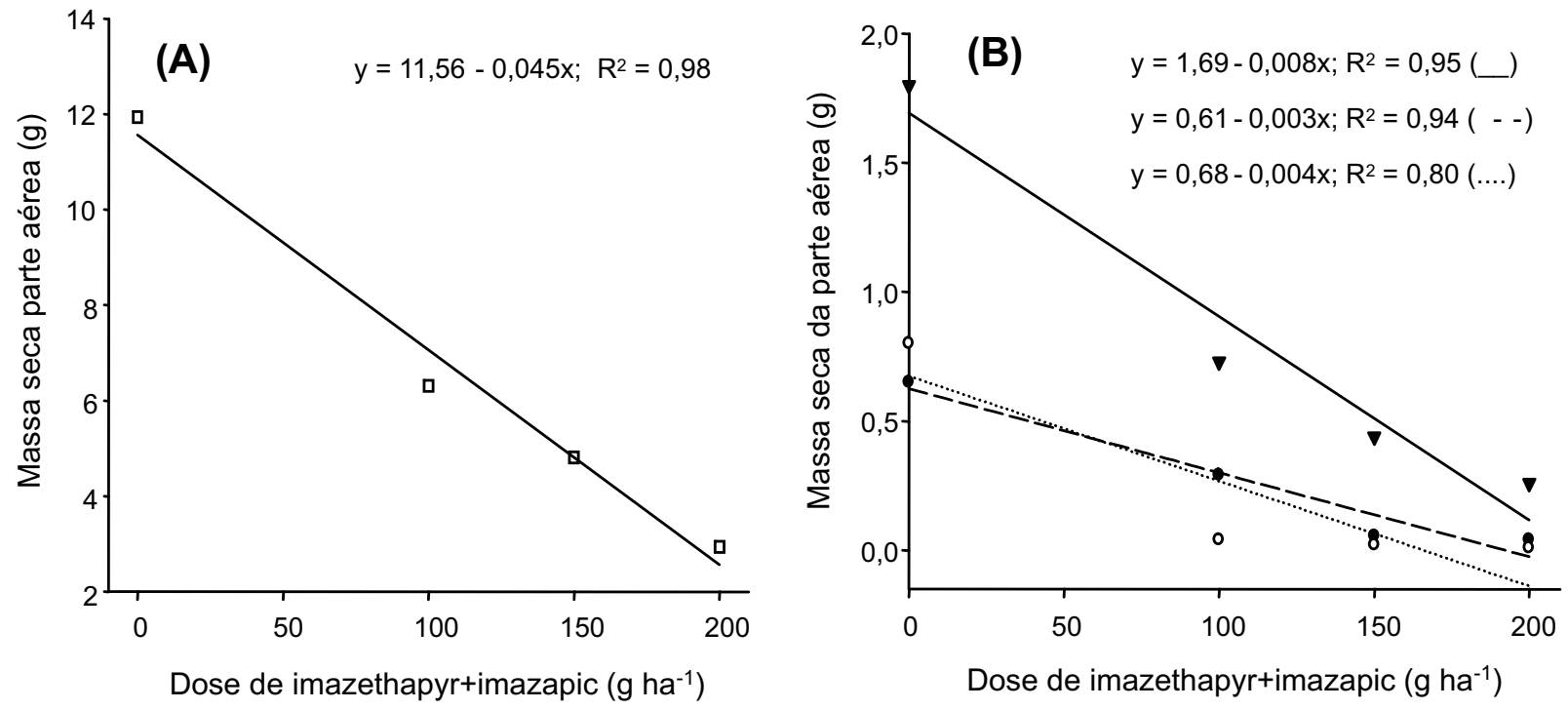

Figura 2 - Efeito residual do herbicida Only ${ }^{\circledR}$ na massa seca da parte aérea de plantas de milho (A e $\square$ ), pepino (_e $\mathbf{\nabla}$ ), rabanete (- - e •) e tomate (....e o) (B) aos 60 dias após a semeadura, cultivados em rotação com o arroz CL, aos 1.100 dias após a aplicação dos herbicidas. Capão do Leão-RS, 2010.

foram observadas reduções de 39, 47, 49 e 59\% da massa seca da parte aérea, respectivamente para as plantas de milho (Figura 2A), pepino, rabanete e tomate (Figura 2B). Esse efeito foi mais acentuado no solo que continha resíduos de $200 \mathrm{~g} \mathrm{ha}^{-1}$, onde as reduções de massa da matéria seca atingiram 78, 95, 98 e $100 \%$, para as plantas de milho (Figura 2A), pepino, rabanete e tomate, respectivamente (Figura 2B). A atividade residual no solo de herbicidas do grupo das imidazolinonas também foi observada por Gazziero et al. (1997), que encontraram, aos 150 dias após a sua aplicação, redução da massa da matéria seca da parte aérea das plantas de milho semeado em solos contendo resíduos de imazaquin e imazethapyr. Alister \& Kogan (2005) também relataram que, de 11 culturas testadas, nove apresentaram redução na produção de massa da matéria seca da parte aérea devido à atividade residual dos herbicidas imazethapyr+ imazapic.

A análise estatística dos dados de massa seca das raízes de tomate e rabanete (Figura 3) demonstrou reduções de 57 e $59 \%$ quando as plantas foram semeadas sobre o residual da dose de $100 \mathrm{~g} \mathrm{ha}^{-1} \mathrm{de} \mathrm{Only}^{\circledR}$, respectivamente para as plantas de rabanete e tomate. A dose de $200 \mathrm{~g} \mathrm{ha}^{-1}$ não foi tolerada pelas plantas, resultando na ausência de raízes. Herbicidas do grupo das imidazolinonas têm mostrado persistência no solo superior a 300 dias para tomateiro (Alister \& Kogan, 2005), com potencial de ocasionar fitotoxicidade à cultura semeada em rotação que não seja tolerante aos herbicidas (Ball et al., 2003).

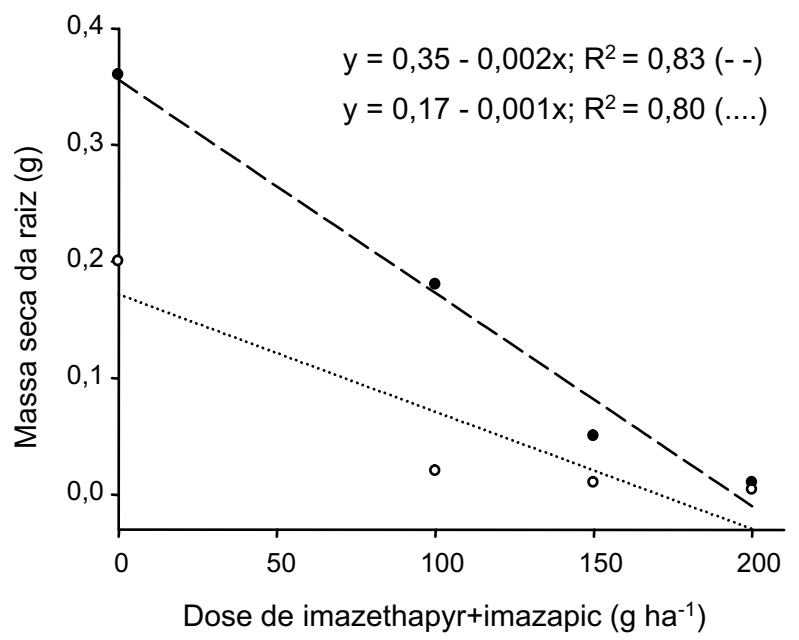

Figura 3 - Efeito residual do herbicida Only ${ }^{\circledR}$ na massa seca da raiz de plantas de rabanete $(--$ e $\bullet$ ) e tomate (....e o) aos 60 dias após a semeadura, cultivados em rotação com o arroz CL, aos 1.100 dias após a aplicação dos herbicidas. Capão do Leão - RS, 2010. 
A sorção desses herbicidas aos coloides do solo é outro fator que interfere na persistência destes e os protege dos processos de biodegradação (Kraemer et al., 2009). Essa sorção é influenciada por diversos fatores, como a temperatura (Flint \& Witt, 1997), a umidade (Curran et al., 1992) e o pH do solo. Baughman \& Shaw (1996) demonstraram que imazethapyr e imazaquin foram mais persistentes em solos frios e secos do que em solos aquecidos e úmidos. Solos com boas condições de umidade promovem dissipação mais rápida dos herbicidas do grupo químico das imidazolinonas (Loux \& Reese, 1993). Essas informações podem justificar a alta persistência dos herbicidas imazethapyr e imazapic encontrada neste experimento, já que nos períodos em que o solo foi mantido sem cobertura não foram realizadas irrigações, fato esse que pode ter aumentado a sorção desses herbicidas no solo, dificultando assim a sua decomposição.

Tendo em vista esses fatores, recomenda-se um intervalo de 300 dias entre a aplicação de herbicidas imidazolinonas e a semeadura do milho em rotação (Rodrigues \& Almeida, 2005). Contudo, esse intervalo poderá ser superior a 1.100 dias, dependendo das condições climáticas e do solo, como observado no presente trabalho.

Os resultados encontrados nesta pesquisa mostraram que:

a) Pode haver presença de atividade residual da mistura comercial dos herbicidas (imazethapyr+imazapic) em solo após 1.100 dias da sua aplicação, podendo essa presença ser detectada através de plantas de milho, pepino, rabanete e tomate.

b) O tomate e o rabanete possuem maior sensibilidade ao produto, quando comparados ao pepino e ao milho.

c) O milho em rotação com a cultura do arroz que utiliza a tecnologia Clearfield ${ }^{\circledR}$ pode sofrer redução na altura e no acúmulo de massa seca da parte aérea das plantas.

\section{AGRADECIMENTOS}

Ao CNPq, à CAPES e à FINEP, pelo suporte financeiro e auxílio com bolsas.

\section{LITERATURA CITADA}

ALISTER, C.; KOGAN, M. Efficacy of imidazolinone herbicides applied to imidazolinone-resistant maize and their carryover effect on rotational crops. Crop Protec., v. 24, n. 4, p. 375-379, 2005.

BALL, D. A.; YENISH, J. P.; ALBY, T. Effect of imazamox soil persistence on dryland rotational crops. Weed Technol., v. 17, n. 1, p. 161-165, 2003.

BAUGHMAN, T. A.; SHAW, D. R. Effect of wetting/drying cycles on dissipation patterns of bioavailable imazaquin.

Weed Sci., v. 44, n. 2, p. 380-382, 1996.

BOND, J. A. et al. Corn and rice response to simulated drift of imazethapyr plus imazapyr. Weed Technol., v. 20, n. 1, p. 113-117, 2006.

CURRAN, W. S. et al. Photolysis of imidazolinone herbicides in aqueous solution and on soil. Weed Sci., v. 40, n. 1, p. 143-148, 1992.

EMPRESA BRASILEIRA DE PESQUISA AGROPECUÁRIA - EMBRAPA. Centro Nacional de Pesquisa de Solos. Sistema brasileiro de classificação de solos. Rio de Janeiro: 2006. 306 p.

FLINT, J. L.; WITT, W. W. Microbial degradation on imazaquin and imazethapyr. Weed Sci., v. 45, n. 4, p. 586-591, 1997.

GAZZIERO, D. L. P. et al. Persistência dos herbicidas imazaquin e imazethapyr no solo e os efeitos sobre plantas de milho e pepino. Planta Daninha, v. 15, n. 2, p. 162-169, 1997.

GOMES JR., F. G.; CHRISTOFFOLETI, P. J. Biologia e manejo de plantas daninhas em áreas de plantio direto. Planta Daninha, v. 26, n. 4, p. 789-798, 2008.

KRAEMER, A. F. et al. Destino ambiental dos herbicidas do grupo das imidazolinonas. Planta Daninha, v. 27, n. 3, p. 629-639, 2009.

LOUX, M. M.; REESE, K. D. Effect of soil type and pH on persistence and carryover of imidazolinone herbicides. Weed Technol., v. 7, n. 2, p. 452-458, 1993.

MACHADO, A. A.; CONCEIÇÃO, A. R. WinStat Sistema de Análise Estatística para Windows versão 1.0. Pelotas: Universidade Federal de Pelotas, 2007.

PINTO, J. O. et al. Milho (Zea mays) como espécie bioindicadora da atividade residual de (imazethapyr + imazapic). Planta Daninha, v. 27, p. 1005-1014, 2009. Número Especial. 
RODRIGUES, B. N.; ALMEIDA, F. S. Guia de herbicidas. 5.ed. Londrina: Edição dos Autores, 2005. 591 p.

SHANER, D. L.; O`CONNOR, S. The imidazolinones herbicides. Boca Raton: CRC Press, 1991. 289 p.

SOCIEDADE SUL BRASILEIRA DE ARROZ IRRIGADO SOSBAI. Arroz irrigado: recomendações técnicas da pesquisa para o Sul do Brasil. Porto Alegre: 2010. 188 p.

STOUGAARD, R. N.; SHEA, P. J.; MARTIN, A. R. Effect of soil type and $\mathrm{pH}$ on adsorption, mobility, and efficacy of imazaquin and imazethapyr. Weed Sci., v. 38, n. 1, p. 67-73, 1990.
VAUGHAN, L. K. et al. Is all red rice found in commercial rice really Oryza sativa? Weed Sci., v. 49, n. 4, p. 468-476, 2001.

WANG, X.; WANG, H.; FAN, D. Degradation and metabolism of imazapyr in soils under aerobic and anaerobic conditions. Intern. J. Environ. Anal. Chem., v. 86, n. 8, p. 541-551, 2006.

WILLIAMS, B. J.; STRAHAN, R.; WEBSTER, E. P. Weed management systems for Clearfield Rice. Louisiana Agric., v. 45, n. 3, p. 16-17, 2002. 